\title{
Inspection of Electric Power Distribution Systems with SCRDet
}

\author{
Guilherme de Moraes Restani \\ Department of Electrical Engineering \\ Federal University of Parana \\ Curitiba, Brazil \\ guilherme.restani@ufpr.br
}

\author{
Leandro dos Santos Coelho \\ Department of Electrical Engineering \\ Federal University of Parana, \\ Industrial and Systems Engineering \\ Graduate Program (PPGEPS) \\ Pontifical Catholic University of Parana \\ Curitiba, Brazil \\ 1scoelho2009@gmail.com
}

\author{
José Francisco Bianchi Filho \\ Department of Electrical Engineering \\ Federal University of Parana, \\ Power Systems \\ Lactec \\ Curitiba, Brazil \\ josefranciscobianchi@gmail.com
}

\begin{abstract}
Faults in power distribution systems are among the factors that most affect the quality and continuity of the electric power supply, leading to higher operational costs and user dissatisfaction. Given this problem, this research applies deep learning techniques to develop an application capable of detecting utility poles in images, their angles, and the presence of fuse cutouts and their status (open or closed). The result is a system that can be used, among other applications, to detect or prevent faults in power distribution systems and as an asset inventory tool. We hypothesized that it is possible to use methods that perform well in the Dataset for Object Detection in Aerial images (DOTA) to accomplish this result, so, through the review of state of the art in object detection, we choose the SCRDet network to implement. Since there was no publicly available dataset with images of poles and fuse cutouts, we built it from scratch. Through the training and test, it was possible to evaluate the results and make adjustments, reaching a satisfactory result that has proven the viability of such application.
\end{abstract}

Index Terms-Power distribution. Inspection. Object detection. Utility pole.

\section{INTRODUCTION}

Electrical energy is essential for the maintenance of human activities and the development of society. Furthermore, electricity, produced and delivered through generation, transmission, and distribution systems, is one of the largest consumer markets [1].

The distribution systems are responsible for about $90 \%$ of consumer reliability problems [1], directly impacting the cost of electricity and customer satisfaction. For that reason, companies must find effective ways to reduce their costs without affecting the quality of the service provided and find new ways to guarantee the continuity of the energy supply to their consumers. In order to offer a reliable service, energy companies turned to the predictive analysis of their systems, observing points likely to cause failure and evaluating strategies to avoid them [2].

Several factors can impact the quality and continuity of electricity distribution. They can originate from equipment failures from manufacturing defects, damages caused during delivery, incorrect installation, aging, and operating conditions.
They can also be caused by animals or vegetation, severe weather conditions, and even human error [1].

In order to ensure the integrity of the power distribution equipment, it is necessary to carry out a visual inspection, which is laborious given the magnitude that this type of system usually has. In addition, another challenge for companies is to keep their inventory of assets updated since there is a considerable number of equipment from different manufacturers installed.

Therefore, this work aims to investigate and begin the development of a tool that allows evaluating the current condition of installed electrical equipment through their detection in images. This stage's result is a tight crop around the detected object, which can be used as an input with less noise for an anomaly detection pipeline. As a result, the inspection process can be accelerated, increasing its level of assertiveness and reliability.

In recent years, the artificial intelligence field is one of the areas of science that has shown significant development, especially its subfield of deep learning, which concerns the development and implementation of algorithms for Artificial Neural Networks (ANNs). Deep learning models have achieved stateof-the-art precision and have been widely applied in several areas. It has also become a popular approach for the task of extracting knowledge from digital images.

Therefore, this project investigates and uses the most recent advances in artificial intelligence, such as Convolutional Neural Networks (CNNs), to develop an application that contributes to the quality and reliability of the electrical energy distribution system.

Two elements were chosen for the initial study: utility poles and fuse cutouts. In the case of utility poles, there is interest in detecting their angles since this may indicate their risk of falling off. In the case of cutout fuses, the aim is to detect their two states, open and closed. These elements were chosen because fuse cutouts and poles have a broad difference in scale, in addition to appearing in different contexts (fuse cutouts usually appear in groups and close to each other). Open and closed fuse cutouts have little visual difference between 
them; that is also the case with many other types of equipment found in distribution systems. Thus, these three cases were chosen as they allow an analysis of distinct scenarios, which reflect the complexity of various configurations and situations in which power distribution equipment can appear.

The remainder of this paper is organized as follows: Section II describes the methodology utilized. Section III shows the results of the literature review. Section IV explains the aspects of the ANN chosen to implement the proposed solution. Section $\mathrm{V}$ describes the construction of a dataset containing images of poles and fuse cutouts. It also shows the results obtained with the implementation of the ANN chosen. Section VI discusses the results, brings the conclusions, and presents further works.

\section{Methods}

This research carried out a literature review and analysis of what has been done on utility poles and fuse cutouts detection. It was also performed research on artificial intelligence, focusing on the applications of object detection in images, by examining surveys and articles on journals and conferences with significant impact published in recent years.

The metric utilized to compare different ANN architectures and evaluate the results obtained in this research was the mean Average Precision (mAP). It allows measuring the classification and localization accuracy of an object detection.

A database was created with images of utility poles and fuse cutouts. Afterward, the data were manipulated to comply with the labeling format used by the network chosen.

Data augmentation techniques were implemented to address the dataset imbalance, and adaptations were made to the chosen algorithm to better model the desired classes.

\section{RELATED WORKS}

Although it is possible to find examples in the literature, there is not a wide range of publications addressing the detection of utility poles in images, let alone ones that approach, specifically, the detection of their angles and components.

One of the first works about the theme that makes use of Computer Vision was [3], which, when dealing with the inspection of overhead power lines using video, also ends up encompassing the detection of utility poles. Later, Jones, Whitworth, and Duller [4] dedicate themselves to improve this application. The authors present an inspection algorithm that, in a rudimentary way, can locate the position of the utility poles using the digital image processing technique known as pattern matching. This technique, however, at the time had a high computational cost, low accuracy and wasn't suitable for different models of utility poles.

Golightly and Jones [5], Golightly and Jones [6] and Jones et al. [7] when approaching the problem of automating the inspection of overhead power lines, propose a detection method for poles based on contour detection, which resulted in an improvement in the task. However, unsatisfactory results were obtained due to the variation in lighting and patterns in the background of the images.
Cheng and Song [8] propose an approach for the detection of poles in images that is based on the graph-cut technique for image segmentation. In this technique, the image is seen as a weighted graph in which the image's pixels represent the nodes. To reduce the computational cost, at first, the image undergoes filtering in which straight lines are detected, a region in which the pole can be found is defined and subsequently, the construction of the weighted graph takes place. At the time, this technique showed an improvement in the results that had been obtained until then.

We can also extrapolate and observe other applications that are related to the problem, such as the detection of poles in aerial images in [9], which uses the detection of the shadow of the poles and techniques such as pattern matching and feature extraction.

Another related problem is the detection of power transmission towers in images [10],[11]. Both works cited make use of the Hough Transform, the former will later employ an infinite impulse response (IIR) filter and the latter applies a data clustering process.

Steiger, Lucas, and Maret [12] however, separate its application into training and testing phases. In training, the extraction of features through the Line Segment Detector, the clustering of data and the obtainment of spatial distributions are performed. In detection, the extraction of image characteristics, the correspondence with the model and a voting process are carried out comparing the values obtained with those of the training model, and thus locating the object of interest.

Still dealing with the detection of electrical energy transmission towers in images, Sampedro et al. [13] and Martinez et al. [14] use an approach that makes use of the Histogram of Oriented Gradients (HOG), for feature extraction, and makes use of the ANN of the type multilayer perceptron.

In addition, there is also an interest in the literature in carrying out the automatic detection of utility poles in urban environments, as we see in [15] that use data pre-processing, feature extraction, support vector machine (SVM) and clustering to obtain the results.

Going back to pole detection, Sharma et al. [16] propose a methodology with a shape-based template that can return results even when the pole is tilted. However, this approach is restricted to only one type of pole - which resembles the template.

The detection of poles is also seen within the theme of autonomous vehicles, as in [17], which uses stereo-vision technique to perform the task.

Already using deep learning techniques Nordeng et al. [18] use the CNN Faster-RCNN, in addition to data augmentation techniques, to perform the detection of dead end body components in power transmission towers.

Making use of images from the Google Street View platform and the one-stage detector RetinaNet, Zhang et al. [19] are able to identify and locate poles with cross-arm on images with good results.

Another application that also makes use of a one-stage network detector is found in the work of Chen and Miao 
[20]. The authors use the You Only Look Once (YOLO) network to perform the detection and counting of towers in transmission lines through images obtained with an unmanned aerial vehicle. Like the previous application, this type of network proves to be advantageous for its ability to perform the detection task quickly.

\section{SCRDET}

The SCRDet (previously called $R^{2} \mathrm{CNN}++$ ) is a multi-class object detector of general-purpose that performs well in the task of detecting objects in aerial image databases, such as the Large-Scale Data Set for Object Detection in Aerial Images (DOTA) [21] and the NWPU VHR-10 [22] datasets. For this reason, it is designed to handle images that present objects of various dimensions, in cluttered and dense arrangements, with arbitrary orientations, and with background noise.

\section{A. Architecture of SCRDet}

In Figure 1 we see the composition of the SCRDet architecture, which can be divided into the three main stages presented below.

The first stage of the network is the SF-Net, which consists of using the ResNet layers $\left(C_{1}, C_{2}, C_{3}\right)$ together with other layers to reduce the set of characteristics that are relevant to the problem.

Next is the multi-dimensional attention network (MDANet), a layer that aims to reduce noise and improve the separation between the information of interest and information that can reduce detection quality.

Lastly is the rotation step. In this block, the network will perform the regression of the bounding boxes and classify the found objects. This process takes place in two stages. In the first stage, a Region Proposal Network (RPN) roughly generates the propositions for the second stage. Next, in the second stage, the parameters $(x, y, w, h, \theta)$ are used, where $\theta$ is in the range $[-\pi / 2,0)$, to denote the rectangle arbitrarily rotated, $x$ and $y$ are the box's center coordinates, and $w$ and $h$ are the box's width, height, respectively. In Figure 2 we observe the attributes of the selection box, which will be used by the network throughout the learning process so that it can perform the regression on objects that present arbitrary rotations. In blue, we have the bounding box proposed for the object; horizontally, in green, we have the rotated selection box from the data annotation; and finally, in red, the selection box inferred by the network.

These parameters compose the cost function used by the network, presented in the Equation 1. Where $N$ is the number of proposed boxes, $t_{n}$ represents the object label, $p_{n}$ is the probability distribution calculated by the Softmax function, $t_{n}^{\prime}$ is a binary value ( 1 for foreground and 0 for background), $v_{* j}^{\prime}$ is the offset predicted vector, $v_{* j}$ is the vector of with the true values from the data annotation, $u_{i j}$ and $u_{* j}^{\prime}$ are the pixels of the true and predicted masks, respectively, and the Intersection over Union (IoU) is the overlap between the true and predicted bounding box. $\lambda_{1}, \lambda_{2}$ and $\lambda_{3}$ are hyperparameters for controlling the trade-off. $L_{c l s}$ is the crossentropy of the Softmax function, $L_{r e g}$ regression loss is the smooth loss $L_{1}$, defined in [23], and $L_{a t t}$ is the cost of the attention function of the cross-entropy of the Softmax concerning the pixel.

$$
\begin{array}{r}
L=\frac{\lambda_{1}}{N} \sum_{n=1}^{N} t_{n}^{\prime} \sum_{j \in\{x, y, w, h, \theta\}} \frac{L_{r e g}\left(v_{n j}^{\prime}, v_{n j}\right)}{\left|L_{r e g}\left(v_{n j}^{\prime}, v_{n j}\right)\right|}|-\log (I o U)|+ \\
\frac{\lambda_{2}}{h \times w} \sum_{i}^{h} \sum_{j}^{w} L_{a t t}\left(u_{i j}^{\prime}, u i j\right)+\frac{\lambda_{3}}{N} \sum_{n=1}^{N} L_{c l s}\left(p_{n}, t_{n}\right)
\end{array}
$$

Throughout its architecture, the SCRDet uses techniques such as RPN, other small convolutional networks to improve the stages, fully connected layers, and activation functions such as sigmoidal and ReLu in addition to the softmax function.

\section{B. Comparative}

To understand how the SCRDet network performs against other methods for rotated object detection, we took into account their mean Average Precision (mAP) achieved on DOTA dataset. This score, which goes from 0 to 1 (being 1 the best possible result), considers the trade-off between precision, recall, and IoU metrics.

In Table I, we have a comparison of the mAP (for an IoU $\geq 0.5$ ) of several two-stage type object detectors, where RRPN is the Rotation Region Proposal Networks, RRPN is the Rotational Region CNN, and ICN is the Image Cascade Network ICN. The best result is achieved by SCRDet with and mAP@0.5 of 72.61 .

Table I

Two-STAGE DeTECTORs - MAP@ 0.5

\begin{tabular}{|c|c|c|c|c|}
\hline $\boldsymbol{R}^{2} \mathbf{C N N}$ & RRPN & ICN & RoI-Trans & SCRDet \\
\hline 60.67 & 61.01 & 68.20 & 69.56 & 72.61 \\
\hline
\end{tabular}

Next, in Table II, we have a comparison of the mAP (for an IoU $\geq 0.5$ ) of several one-stage type object detectors. The best result is achieved by $\mathrm{R}^{3}$ Det+ResNet152 with and mAP@0.5 of 72.81 .

Table II

ONE-STAGE DETECTORS - MAP@ 0.5

\begin{tabular}{|c|c|c|c|}
\hline SSD & YOLOv2 & $\boldsymbol{R}^{\mathbf{3}}$ Det+ResNet101 & $\boldsymbol{R}^{\mathbf{3}}$ Det+ResNet152 \\
\hline 17.84 & 25.49 & 71.69 & 72.81 \\
\hline
\end{tabular}

Since the SCRDet and R3Det+ResNet152 present close results in these comparisons, it was decided to use the SCRDet [24] network, given that two-stage object detectors frequently show more robustness in tasks of object detection [25].

\section{DeVelopment AND RESUlts}

This section presents the results obtained using the SCRDet network with our dataset. We used the following code [26], available on GitHub, as reference. 


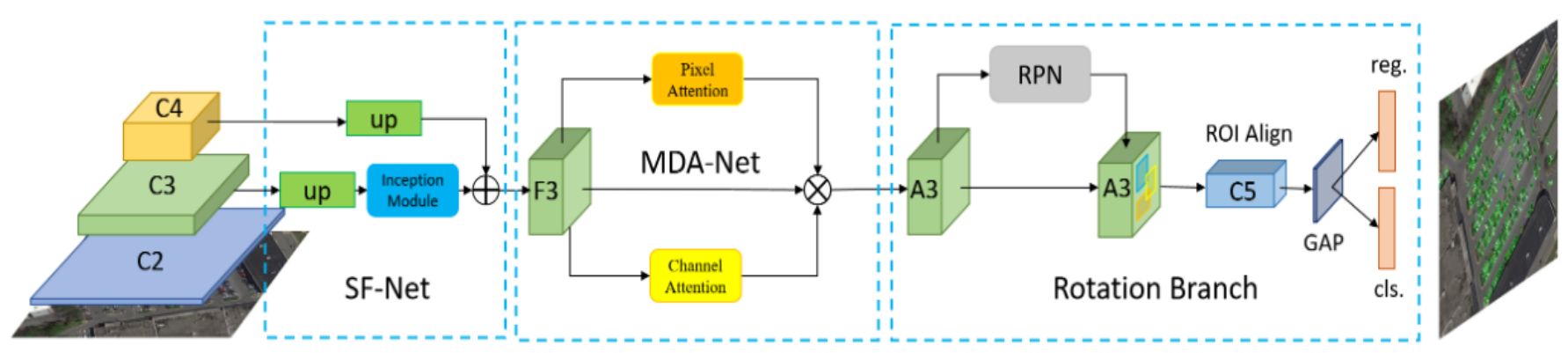

Figure 1. SCRDet network architecture.

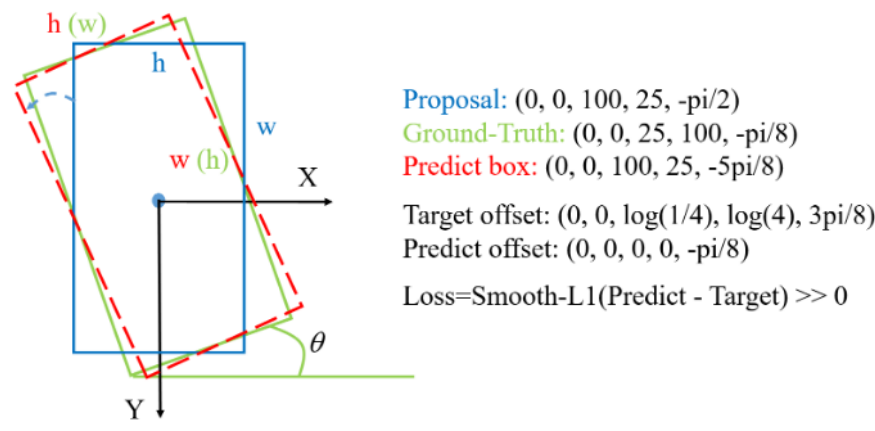

Figure 2. Representation of the selection boxes used by the SCRDet network.

\section{A. Development Environment Settings}

The equipment used to execute the code was a microcomputer with an Intel Core i7-9700k processor, 32GB of randomaccess memory (RAM), and a GeForce RTX 2080 Ti graphics card.

We used the Python programming language, OpenCV for image processing, and TensorFlow to implement the neural networks. Also, we used the Compute Unified Device Architecture (CUDA) 8.0 parallel processing Application Programming Interface (API) running on Linux Ubuntu 18.04 distribution.

\section{B. Dataset}

It was not possible to verify the existence of a public dataset containing images of the objects of interest. Therefore, it was necessary to create a new database from scratch. For this purpose, 2252 images were acquired through the internet and from field records made by us. The aim was to represent the broadest possible range of scenarios, including variations in climate, vegetation, and urbanization. The images that compose the database are in different orientations and resolutions. Figure 3 shows image clipping samples from the database created.

The images were shuffled and divided into training and test, with partitions of $80 \%$ and $20 \%$, respectively. Table III shows how objects of each class appear in the data partitions.

The database presented a class imbalance, with the utility pole being the most frequent and the fuse cutout in its open state the least frequent. In order to balance the dataset,

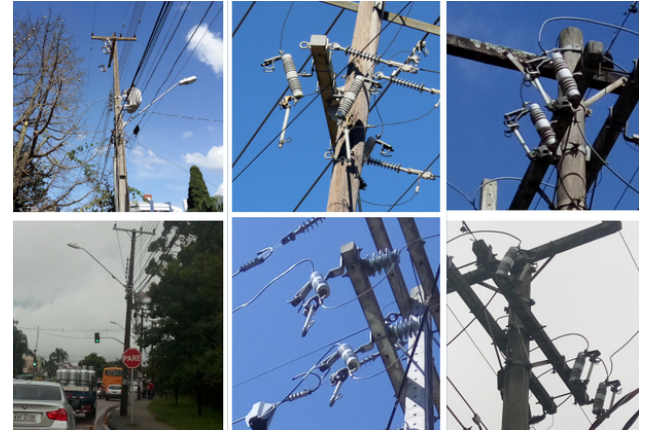

Figure 3. Example of images from the utility poles and fuse cutouts database.

Table III

COMPOSITION OF TRAINING AND TESTS SETS

\begin{tabular}{|c|c|c|c|}
\hline Class & Quantity & Training & Test \\
\hline Pole & 2210 & 1754 & 456 \\
\hline Fuse cutout close & 1953 & 1579 & 374 \\
\hline Fuse cutout open & 799 & 627 & 172 \\
\hline Total & 4962 & 3960 & 1002 \\
\hline
\end{tabular}

images of the pole class were excluded, and data augmentation techniques were applied to the open fuse cutouts set. Those operations were zoom, brightness, Gaussian noise, shift, horizontal flip, and random rotation between 0 to 10 degrees.

\section{Execution}

The training was executed with a single image batch, given the hardware limitations, and an initial learning rate of $3 \times 10^{-4}$, which decreased to $3 \times 10^{-5}$ in the 150,000 th iteration and $3 \times 10^{-6}$ in the 250,000th iteration. This strategy aims to move faster towards the minimum of the cost function in the initial moments and, subsequently, to promote a finer adjustment of this value.

After training up to the 450,000th iteration, the network was evaluated using the test set. The metric chosen to evaluate the model's performance was the mAP (mean Average Precision) with an IoU of 0.5. The results can be seen in the Figure 4, next, which shows the curve of precision on the $y$-axis, by the recall on the $x$-axis.

By smoothing and measuring the area under the graph for each class, we obtained the results presented in Table IV. The resulting mAP score is $0.7338 @ 0.5$. 


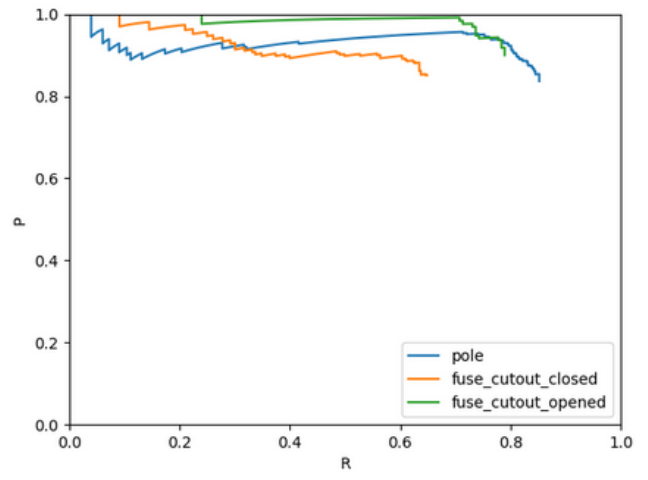

Figure 4. mAP @ 0,5: in blue, class pole; in orange, class closed cutout fuse; and in green, class open cutout fuse.

Table IV

RESULTS

\begin{tabular}{|c|c|}
\hline Class & Average Precision \\
\hline Pole & 0.8122 \\
\hline Fuse cutout closed & 0.6076 \\
\hline Fuse cutout opened & 0.7818 \\
\hline mAP @ 0.5 & 0.7338 \\
\hline
\end{tabular}

The inference performed on the test partition, which sample results can be seen in Figure 5, took an average of $200 \mathrm{~ms}$ for each image.
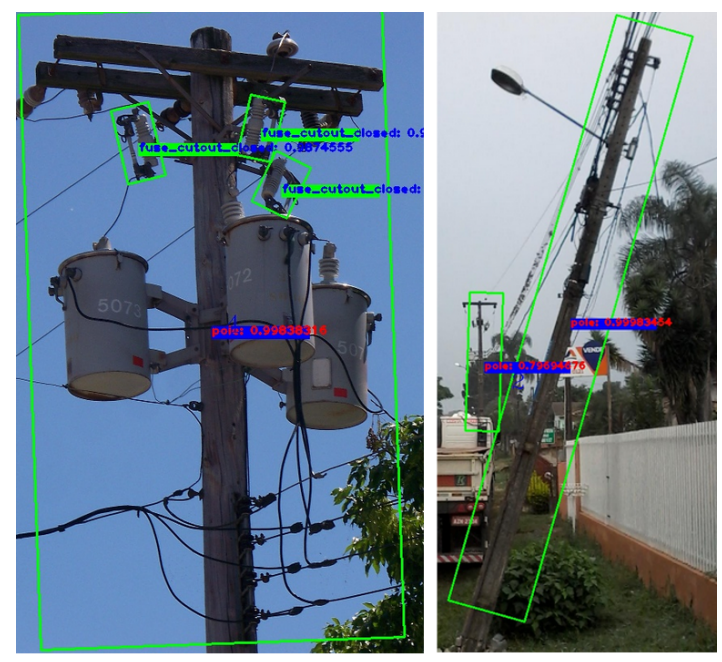

Figure 5. Sample of inference results.

\section{CONCLUSION}

Although little specific content has been developed on power distribution equipment detection, the literature review could show an evolution from classical machine learning methods towards deep learning approaches. It also gave insight into how CNNs could be used to address the problem presented in this paper.

Next, we decided to use a network developed for detecting rotated objects in aerial images. Given their characteristics, they can detect objects in cluttered and dense arrangements with a lot of background noise. Similarly, fuse cutouts usually appear in this type of configuration.

A public domain database with images of power distribution equipment was not found. Therefore, we built a dataset containing poles and fuse cutouts from scratch. This step proved to be challenging given the amount of labor involved in the image acquisition and data labeling processes.

We used deep learning to detect utility poles in images instead of digital image processing techniques, such as in [8] and [16]. In the last years, this approach became popular for object detection given its robustness and more flexibility compared to traditional image processing methods. Zhang et al. [19], also use deep learning, which suits well their purpose of mapping utility poles, but our approach offers a better object crop for a detection pipeline. As there is no reference dataset, the results could not be quantitatively compared with the work of those authors. However, the mAP scores and the visual results obtained showed that using deep learning is a viable approach to the problem defined in the first section of this paper.

The resulting oriented bounding boxes end up being tighter around the objects than the usual horizontal ones. That way, a better crop of the objects can be done with less noise and more meaningful information. Later, this information can feed object detection pipelines constructed to differentiate equipment from different manufacturers, detect anomalies such as rusting, rotting, and more.

Future works include the continuous expansion of the dataset, including the addition of other classes, such as transformers, switches, cross-arms, and insulators, which expand the scope of application possibilities of the system developed in this paper. The dataset first needs to undergo anonymization to be published since there are images in which car plates and people's faces appear.

Another future work is the development of a pipeline that takes the results of the presented implementation to further detect anomalies in power distribution equipment. This pipeline then can be embedded in an image capture system, including a georeferencing system and gimbal (to ensure images are always taken leveled) and later be implemented in vehicles. That way, energy companies can use it to inspect their assets and improve the reliability of their systems.

\section{REFERENCES}

[1] R. Brown. Electric Power Distribution Reliability, Second Edition. Vol. 20081087. Power Engineering (Willis). CRC Press, 2008, p. 504.

[2] A. Guimarães. Confiabilidade de Sistemas de Distribuição: Calibração de Dados e Mecanismos para Avaliação de Desempenho. 2006.

[3] D. I. Jones. "Aerial inspection of overhead power lines using video: Estimation of image blurring due to vehicle and camera motion". In: IEE Proceedings: Vision, Image and Signal Processing 147.2 (2000), pp. 157-166. 
[4] D. I. Jones, C. C. Whitworth, and A. W. G. Duller. "Image processing methods for the visual location of power line poles." In: Proc 7th Irish Machine vision and Image Processing conference (IMVIP). 2003, pp. 177184.

[5] I. Golightly and D. Jones. "Corner detection and matching for visual tracking during power line inspection". In: Image and Vision Computing 21.9 (2003), pp. 827-840.

[6] I. Golightly and D. Jones. "Visual control of an unmanned aerial vehicle for power line inspection". In: 2005 International Conference on Advanced Robotics (ICAR), Proceedings. 2005, pp. 288-295.

[7] D. I. Jones et al. "A laboratory test-bed for an automated power line inspection system". In: Control Engineering Practice 13.7 (2005), pp. 835-851.

[8] W. Cheng and Z. Song. "Power pole detection based on graph cut". In: Proceedings - 1st International Congress on Image and Signal Processing (CISP). Vol. 3. 2008, pp. 720-724.

[9] B. Cetin, M. Bikdash, and M. McInerney. "Automated electric utility pole detection from aerial images". In: Conference Proceedings - IEEE SOUTHEASTCON. 2009, pp. 44-49.

[10] J. Tilawat, S. Auephanwiriyakul, and N.n TheeraUmpon. "Automatic detection of electricity pylons in aerial video sequences". In: ICEIE - International Conference on Electronics and Information Engineering, Proceedings. Vol. 1. 2010.

[11] Z. Li et al. "Knowledge-based power line detection for UAV surveillance and inspection systems". In: $23 \mathrm{rd}$ International Conference Image and Vision Computing New Zealand (IVCNZ). 2008.

[12] O. Steiger, E. Lucas, and Y. Maret. "Automatic detection of transmission towers". In: Proceedings of IEEE Sensors. December. 2014, pp. 1034-1037.

[13] C. Sampedro et al. "A supervised approach to electric tower detection and classification for power line inspection". In: Proceedings of the International Joint Conference on Neural Networks. 2014, pp. 1970-1977.

[14] C. Martinez et al. "Towards autonomous detection and tracking of electric towers for aerial power line inspection". In: International Conference on Unmanned Aircraft Systems (ICUAS) - Conference Proceedings. 2014, pp. 284-295.

[15] F. Tombari et al. "Automatic detection of pole-like structures in 3D urban environments". In: IEEE International Conference on Intelligent Robots and Systems. 2014, pp. 4922-4929.

[16] H. Sharma et al. "Image Analysis-Based Automatic Utility Pole Detection for Remote Surveillance". In: International Conference on Digital Image Computing: Techniques and Applications (DICTA). 2015.

[17] B. C. Z. Blaga and S. Nedevschi. "A method for automatic pole detection from urban video scenes using stereo vision". In: Proceedings - IEEE 14th Interna- tional Conference on Intelligent Computer Communication and Processing (ICCP). 2018, pp. 293-300.

[18] I. E. Nordeng et al. "DEBC detection with deep learning”. In: Lecture Notes in Computer Science (including subseries Lecture Notes in Artificial Intelligence and Lecture Notes in Bioinformatics). Vol. 10269 LNCS. 2017, pp. 248-259.

[19] W.g Zhang et al. "Using deep learning to identify utility poles with crossarms and estimate their locations from google street view images". In: Sensors (Switzerland) 18.8 (2018).

[20] B. Chen and X. Miao. "Distribution Line Pole Detection and Counting Based on YOLO Using UAV Inspection Line Video". In: Journal of Electrical Engineering \& Technology 15.1 (2020), pp. 441-448.

[21] G. S. Xia et al. "DOTA: A Large-Scale Dataset for Object Detection in Aerial Images". In: Proceedings of the IEEE Computer Society Conference on Computer Vision and Pattern Recognition. 2018, pp. 3974-3983.

[22] G. Cheng et al. "Multi-class geospatial object detection and geographic image classification based on collection of part detectors". In: ISPRS Journal of Photogrammetry and Remote Sensing (2014).

[23] R. Girshick. "Fast R-CNN". In: Proceedings of the IEEE International Conference on Computer Vision. 2015, pp. 1440-1448.

[24] X. Yang et al. "R ${ }^{3}$ Det: Refined Single-Stage Detector with Feature Refinement for Rotating Object". In: arXiv preprint arXiv:1908.05612 (2019).

[25] Z. Zou et al. "Object Detection in 20 Years: A Survey". In: arXiv preprint arXiv:1905.05055 (2019).

[26] X. Yang and J. Yang. R2CNN-Plus-Plus_Tensorflow, (2019). GitHub repository, https : / / github . com / DetectionTeamUCAS/R2CNN-Plus-Plus_Tensorflow. 